\title{
AN ADAPTIVE NONPARAMETRIC \\ CONTROLLER FOR A CLASS OF NONMINIMUM PHASE NON-LINEAR SYSTEM
}

\author{
Daniel Sbarbaro. * Roderick Murray-Smith. ${ }^{* *}$ \\ * Universidad de Concepción,Concepción, CHILE. \\ http: \\www.die.udec.cl. \\ ** Department of Computing Science, University of \\ Glasgow Scotland \\ $\&$ Hamilton Institute, NUI Maynooth, Ireland \\ http: \\www.dcs.gla.ac.uk ${ }^{\sim}$ rod
}

\begin{abstract}
This paper addresses the problem of controlling a class of unknown nonlinear systems with unstable inverse, by using a generalized minimum variance approach. We highlight the limitations of the classic cost function used in this context and we propose a new one based on the Generalized Feedback Linearization concept. The adaptive control algorithm uses a nonparametric model, which provides an estimate of the mean and variance of the system output. Simulation examples illustrate the main characteristics and limitation of the proposed approach. Copyright ${ }^{\circledR} 2005$ IFAC.
\end{abstract}

Keywords: Adaptive systems, Discrete non-linear system, Generalized Minimum Variance, non-parametric models.

\section{INTRODUCTION}

Adaptive control of discrete nonlinear systems using flexible nonlinear parameterization like Artificial Neural Networks (Jagannathan and Lewis, 1996; Chen and Khalil, 1995; Narendra and Parthasarathy, 1990) and nonparametric models (Murray-Smith and Sbarbaro, 2002; Portier and Oulidi, 2000; Kocijan et al., 2004) have received some attention. Most of these works have relied on the assumption that the system has stable inverse, even though in discrete systems, nonminimum phase behavior can even be brought by the sampling rate selection, as in the linear case.

(Talebi et al., 2000) describes an output redefinition strategy which is only applicable to a class of open-loop stable nonminimum phase systems. A more general approach based on the Inter- nal Model theory is presented in (Aoyama et al., 1996), but as in the previous case it is only applicable to open-loop stable systems. In (Yue et al., 1997) and (Zhu et al., 1999) a method based on a model which has linear and a residual nonlinear part is proposed, the main underlying assumption is that the nonlinear part has no hidden nonminimum phase effect. This assumption can only be met if the nonminimum phase effect is due to a linear system, limiting its application to many real systems.

The Generalized Minimum Variance control strategy for nonlinear system has been proposed by several authors (Bittanti and Piroddi, 1997; Yue et al., 1997; Liang and ElMargahy, 1994) and more recently (Zhu and Guo, 2004). This strategy provides several degrees of freedom for tackling the control of different systems. However, it can 
be demonstrated that, unlike in the linear case, this extra flexibility is not enough to deal with nonlinear inverse dynamics. A simple additional enhancement, based on the idea of Generalized Feedback Linearization (Goodwin et al., 2001) can be included to deal with a broader class of systems, having nonlinear inverse dynamic. These ideas are used in the framework of nonparametric adaptive controllers (Murray-Smith and Sbarbaro, 2002) for expanding the range of systems which can be controlled by this approach without increasing its complexity.

This work is organized as follows: section 2 describes the limitation of the GMV strategy and proposes an enhanced cost function which also considers the error between the desired control action and the one applied to the process. Section 3 describes a nonparametric modelling approach for modelling the system and the model uncertainty, and in section 4 two controllers are derived from different cost functions. Section 5 illustrates, by simulation examples, the advantages and limitations of the proposed control approach.

\section{GENERALIZED MINIMUM VARIANCE CONTROLLERS}

The objective of this paper is to control a singleinput, single-output, affine nonlinear system of the form,

$$
y(t+d)=f(\mathbf{x}(t))+g(\mathbf{x}(t)) u(t)+\varepsilon(t+d),
$$

where $d$ is defined as the relative degree of the system (Chen and Khalil, 1995), $\mathbf{x}(t)$ is the state vector at a discrete time $t$, which in this paper will be defined as $\mathbf{x}(t)=[y(t), \ldots, y(t-n), u(t-$ $1), \ldots, u(t-m)], y(t+1)$ the output, $u(t)$ the current control vector, $f$ and $g$ are unknown smooth nonlinear functions. A special case is represented by the following equation:

$y(t+d)=f(\mathbf{x}(t))+\sum_{i=0}^{m} g_{i}(\mathbf{x}(t)) u(t-i)+\varepsilon(t+d)$,

where $\mathbf{x}(t)$ is the state vector defined as $\mathbf{x}(t)=$ $[y(t), \ldots, y(t-n)]$. We also assume that $g_{0}$ and $g$ are bounded away from zero. This type of system represents for instance Takagi-Sugeno type of models (Ying, 1999).

The noise term $\varepsilon(t)$ is assumed zero mean Gaussian, but with unknown variance $\sigma_{n}^{2}$. The GMV control strategy consists in choosing a control variable $u(t)$ to minimize the following cost function:

$$
\begin{aligned}
J_{1}= & E\left\{\left(y_{d}(t+d)-y(t+d)\right)^{2}\right\} \\
& +\left(R\left(q^{-1}\right) u(t)\right)^{2},
\end{aligned}
$$

where $y_{d}(t)$ is a bounded reference signal, the polynomial $R\left(q^{-1}\right)$ is defined as:

$$
R\left(q^{-1}\right)=r_{0}+r_{1} q^{-1}+\ldots+r_{n_{r}} q^{-n_{r}}
$$

where $q^{-1}$ is a unit backward shift operator. The polynomial coefficients can be used as tuning parameters.

Using the fact that $\operatorname{Var}\{y\}=E\left\{y^{2}\right\}-\mu_{y}^{2}$, where $\mu_{y}=E\{y\}$, the cost function can be written as:

$$
\begin{aligned}
J_{1}= & \left(y_{d}(t+d)-E\{y(t+d)\}\right)^{2}+ \\
& \operatorname{Var}\{y(t+1)\}+\left(R\left(q^{-1}\right) u(t)\right)^{2} .
\end{aligned}
$$

An enhanced version of the GMV controller can be obtained by considering the idea of Generalized linearizing controller proposed (Goodwin et al., 2001). The cost function represents a combination of two terms:

$$
\begin{aligned}
J_{2}= & (1-\lambda) E\left\{\left(y_{d}-y(t+d)\right)^{2}\right\}+ \\
& \lambda\left(R\left(q^{-1}\right) u(t)-u_{d}\right)^{2}
\end{aligned}
$$

where $u_{d}$ is the input associated to $y_{d}$, the coefficients of $R\left(q^{-1}\right)$ and $\lambda$ are tuning parameters. Clearly when $\lambda=0$ the cost function corresponds to the minimum variance cost function, and when $\lambda=1$ the minimum of the cost function corresponds to a simple feedforward control. If the system is only open loop unstable then $\lambda=0$ can stabilize the system. On the other hand if the open loop system is stable but with unstable inverse, then naturally $\lambda=1$ will provide a stable controller. Fortunately, many real systems can be included in these two categories. Summarizing, the parameter $\lambda$ provides the possibility to balance the combination of a feedforward and feedback control. For systems described by Takagi-Sugeno type of models, equation (2), a simple expression can be obtained for $u_{d}$ :

$$
u_{d}=\frac{y_{d}-f\left(\mathbf{x}_{d}\right)}{\sum_{i=0}^{m} g_{i}\left(\mathbf{x}_{d}\right)}
$$

where $\mathbf{x}_{d}=\left[y_{d}, \ldots, y_{d}\right]$.

\section{A NONPARAMETRIC MODEL}

Let us consider the output of the system be described as:

$$
y_{j}(t+1)=F(\phi(t))+\epsilon_{i}(t)
$$

where $\phi(t)^{T}=\left[\begin{array}{ll}\mathbf{X}^{T}(t) & \mathbf{U}^{T}(t)\end{array}\right]^{T}$. Instead of parameterising the system (1) as a parametric model, we can place a prior directly on the space of functions where $F$ is assumed to belong. A Gaussian process represents the simplest form of prior over functions, we assume that any $p$ points have a $p$ dimensional multivariate Normal distribution. Let the input and output sequence be stacked in the 
following matrix $\Phi_{N}$ and vector $\mathbf{y}_{N}$, respectively. We will assume zero mean, so for the case with partitioned data $\mathbf{y}_{N}$ and $y(t+1)$ we will have the multivariate Normal distribution,

$$
\left[\begin{array}{c}
\mathbf{y}_{N} \\
y(t+1)
\end{array}\right] \sim \mathcal{N}\left(0, \mathbf{C}_{N+1}\right), \quad \mathbf{C}_{N+1}=\left[\begin{array}{cc}
\mathbf{C}_{N} & \mathbf{k} \\
\mathbf{k}^{T} & \kappa
\end{array}\right] .
$$

where $\mathbf{C}_{N+1}$ is the full covariance matrix. Like the Gaussian distribution, the Gaussian Process is fully specified by a mean and its covariance function, so we denote the distribution $G P(\mu, C)$. The covariance function $C(\phi(t), \phi(l))$ expresses the expected covariance between $y(t+1)$ and $y(l+1)$. We can therefore, infer $y(l+1)$ 's from the $N$ data pairs $\Phi_{N}$ and $\mathbf{y}_{N}$ 's rather than building explicit parametric models. The values of the mean and variance of $y(t+1)$ are:

$$
\begin{aligned}
\hat{\mu}_{y} & =\mathbf{k}^{T} \mathbf{C}_{N}^{-1} \mathbf{y}_{N} \\
\operatorname{Var}\{y\}=\hat{\sigma}_{y}^{2} & =\kappa-\mathbf{k}^{T} \mathbf{C}_{N}^{-1} \mathbf{k},
\end{aligned}
$$

so we can use $\hat{\mu}(\phi(t))$ as the expected model output, with a variance of $\hat{\sigma}(\phi(t))^{2}$. In order to simplify notation the following variables are defined:

$$
\begin{aligned}
\omega_{0}= & C_{x}\left(\mathbf{x}(t), \Phi_{N}, \Theta_{x}\right)^{T} \mathbf{C}_{N}^{-1} \mathbf{y}_{N} \\
\omega_{1}= & \left(C_{x}\left(\mathbf{x}(t), \Phi_{N}, \Theta_{u}\right) \cdot * U_{N}\right)^{T} \mathbf{C}_{N}^{-1} \mathbf{y}_{N} \\
\gamma_{0}= & C_{x}\left(\mathbf{x}(t), \mathbf{x}(t), \Theta_{x}\right)- \\
& C_{x}\left(\mathbf{x}(t), \Phi_{N}, \Theta_{x}\right)^{T} \mathbf{C}_{N}^{-1} C_{x}\left(\mathbf{x}(t), \Phi_{N}, \Theta_{x}\right) \\
\gamma_{1}= & -2\left(C_{x}\left(\mathbf{x}(t), \Phi_{N}, \Theta_{u}\right) \cdot * U_{N}\right)^{T} \mathbf{C}_{N}^{-1} C_{x}\left(\mathbf{x}(t), \Phi_{N}, \Theta_{x}\right) \\
\gamma_{2}= & C_{x}\left(\mathbf{x}(t), \mathbf{x}(t), \Theta_{u}\right)- \\
& \left(C_{x}\left(\mathbf{x}(t), \Phi_{N}, \Theta_{u}\right) \cdot * U_{N}\right)^{T} \mathbf{C}_{N}^{-1}\left(C_{x}\left(\mathbf{x}(t), \Phi_{N}, \Theta_{u}\right) * U_{N}\right),
\end{aligned}
$$

where $*$ represents elementwise matrix multiplication. These new variables in turn lead to these new expressions:

$$
\begin{aligned}
\hat{\mu}_{y}= & \omega_{0}(\mathbf{x}(t))+\omega_{1}(\mathbf{x}(t)) u(t) \\
\operatorname{Var}\{y\}= & \gamma_{0}(\mathbf{x}(t))+\gamma_{1}(\mathbf{x}(t)) u(t)+ \\
& \gamma_{2}(\mathbf{x}(t)) u(t)^{2}
\end{aligned}
$$

Since the output is an affine function of the control input, it is reasonable to propose a covariance function with a contribution from control inputs as an affine function as well:

$$
\begin{aligned}
K(\phi(i), \phi(j) ; \Theta)= & C_{x}\left(\mathbf{x}(i), \mathbf{x}(j) ; \Theta_{x}\right)+ \\
& u(i) C_{x}\left(\mathbf{x}(i), \mathbf{x}(j) ; \Theta_{u}\right) u(j)
\end{aligned}
$$

where the first term represents the contribution of the state vector and the second one the contribution of the input signal.

The covariance function for $C_{x}$ represents a straightforward covariance function, as used in (Williams, 1998), which has been empirically demonstrated to work well in practice:

$$
\begin{aligned}
C_{x}(\mathbf{x}(i), \mathbf{x}(j) ; \Theta)= & v_{0} e^{-\frac{1}{2} \sum_{k=1}^{p} \alpha_{k}\left(x_{k}(i)-x_{k}(j)\right)^{2}} \\
& +a_{0}
\end{aligned}
$$

so that the parameter vector $\Theta=\log \left[v_{0}, \alpha_{1, . . p}, a_{0}\right]^{T}$ (the $\log$ is applied elementwise) and $p$ is the dimension of vector $\mathbf{x}$. The parameters are defined to be the log of the variable in equation (11) since these are positive scale-parameters.

The covariance function will also be often viewed as being the combination of a covariance function due to the underlying model $K$ and one due to measurement noise $C_{n}$. The entries $i j$ of this matrix are then:

$$
\mathbf{C}_{N_{i j}}=K\left(\Phi_{N_{i}}, \Phi_{N_{j}} ; \Theta\right)+C_{n}\left(\Phi_{N_{i}}, \Phi_{N_{j}} ; \Theta_{n}\right)
$$

where $\Theta$ denotes a set of parameters, which in the GP framework are also called hyperparameters. It is convenient to specify priors in terms of the hyperparameters, which then can be adapted as the model is fit to the identification data. The covariance associated to the noise $C_{n}()$ could be $\delta_{i j} \mathcal{N}\left(\Phi_{N} ; \Theta_{n}\right)$, which would be adding a noise model $\mathcal{N}$ to the diagonal entries of $\mathbf{C}_{N}$. This framework allows to easily include different noise models, as discussed in (Murray-Smith and Girard, 2001).

The Gaussian Process approach to regression is simple to implement. The disadvantage is its computational complexity, as estimating the mean $\hat{\mu}_{y}$ requires a matrix inversion of the $N \times N$ covariance matrix, which becomes troublesome for identification data where $N>1000$; however, there are efficient techniques to deal with this issue (Seeger et al., 2003), and for dynamic systems, data close to equilibrium can often be summarised as linearisations which can be interpreted as 'derivative observations' leading to a much more efficient implementation of the Gaussian Process prior (Solak et al., 2003).

The hyperparameter vector $\Theta$ provides flexibility to define a family of covariance functions which provide suitable prior distributions over functions. In most cases we will only have uncertain knowledge of $\Theta$. Given unknown hyperparameters we can use numerical methods such as standard gradient-based optimisation tools to optimise hyperparameters.

The log-likelihood $l$ of the training data can be calculated analytically as (Williams, 1998) :

$$
l=-\frac{1}{2} \log \operatorname{det} \mathbf{C}_{N}-\frac{1}{2} \mathbf{y}_{N}^{T} \mathbf{C}_{N}^{-1} \mathbf{y}_{N}-\frac{n}{2} \log 2 \pi .
$$

The partial derivative of the log-likelihood with respect to the hyperparameters is:

$$
\frac{\partial l}{\partial \theta_{i}}=-\frac{1}{2} \operatorname{tr}\left[\mathbf{C}_{N}^{-1} \frac{\partial \mathbf{C}_{N}}{\partial \theta_{i}}\right]+\frac{1}{2} \mathbf{y}_{N}^{T} \mathbf{C}_{N}^{-1} \frac{\partial \mathbf{C}_{N}}{\partial \theta_{i}} \mathbf{C}_{N}^{-1} \mathbf{y}_{N} .
$$


Given $l$ and its derivative with respect to $\theta_{i}$ it is straightforward to use an efficient optimization program in order to obtain a local maximum of the likelihood.

\section{THE CONTROLLERS}

Taking the partial derivatives of the variance and the mean expressions, replacing their values in (3), and minimizing with respect to $u(t)$ it follows:

$$
u(t)=\frac{\left(y_{d}(t+d)-\omega_{0}\right) \omega_{1}+\gamma_{1}-r_{0} \bar{R}\left(q^{-1}\right) u(t)}{r_{0}^{2}+\gamma_{2}+\omega_{1}^{2}}
$$

where $\bar{R}\left(q^{-1}\right)=R\left(q^{-1}\right)-r_{o}$.

In the same way, for the cost function defined as (5), we obtain:

$$
\begin{aligned}
u(t)= & \frac{(1-\lambda)\left[\left(y_{d}(t+d)-\omega_{0}\right) \omega_{1}+\gamma_{1}\right]}{\lambda r_{0}^{2}-(1-\lambda)\left[\gamma_{2}+\omega_{1}^{2}\right]}- \\
& \frac{\lambda r_{0}\left(\bar{R}\left(q^{-1}\right) u(t)-u_{d}\right)}{\lambda r_{0}^{2}+(1-\lambda)\left[\gamma_{2}+\omega_{1}^{2}\right]}
\end{aligned}
$$

If we had not included the variance term in cost functions, or if we were in a region of the statespace where the variance was zero, the optimal control law would be equation (14) or (15) with $\gamma_{1}=\gamma_{2}=0$. These terms make a control effort penalty constant, or regulariser unnecessary in many applications.

\section{SIMULATION RESULTS}

\subsection{Nonlinear system 1 - linear inverse dynamics}

The first nonlinear system represents a discrete time system with linear inverse dynamics described by the following equations:

$f(\mathbf{x}(t))=\frac{y(t) y(t-1)(y(t-2)+1)}{1+y(t-1)^{2}+y(t-2)^{2}}+2.8 u(t-1)$ $g(\mathbf{x}(t))=1$,

where $\mathbf{x}=[y(t) y(t-1) y(t-2) u(t-1)]^{T}$. The polynomial $R\left(q^{-1}\right)$ in the cost function (3) was selected as $R\left(q^{-1}\right)=2.1-2.1 q^{-1}$; by simple inspection it can be seen that these parameters are enough for stabilizing the nominal inverse dynamic. The results obtained for the nominal system are shown in Figure 1. Next, we consider the system as unknown and a system noise with a variance $\sigma_{n}^{2}=0.001$. The adaptive controller described by equation (14) is able, after some transient, to bring the system to the set-point by a bounded control signal, as seen in figure 2 . In this simulation, the controller considered just 10 initial data points.
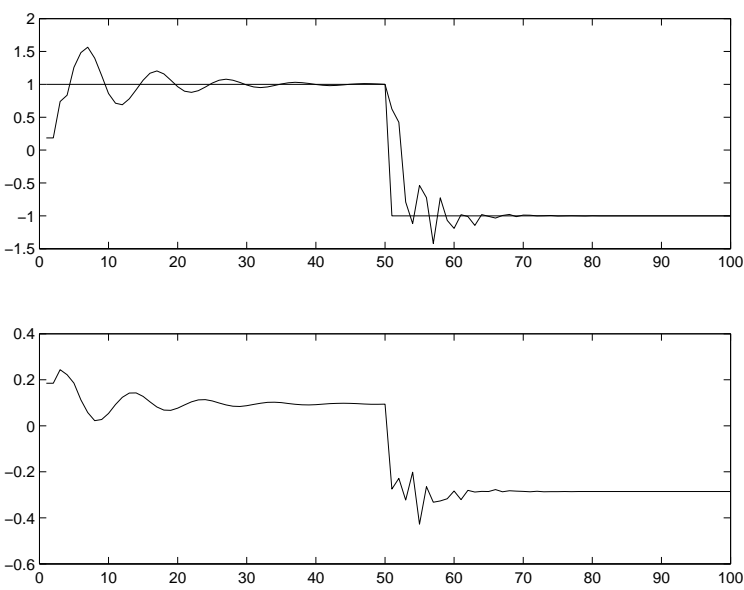

Fig. 1. Control of 1st nonlinear system with linear inverse dynamics.
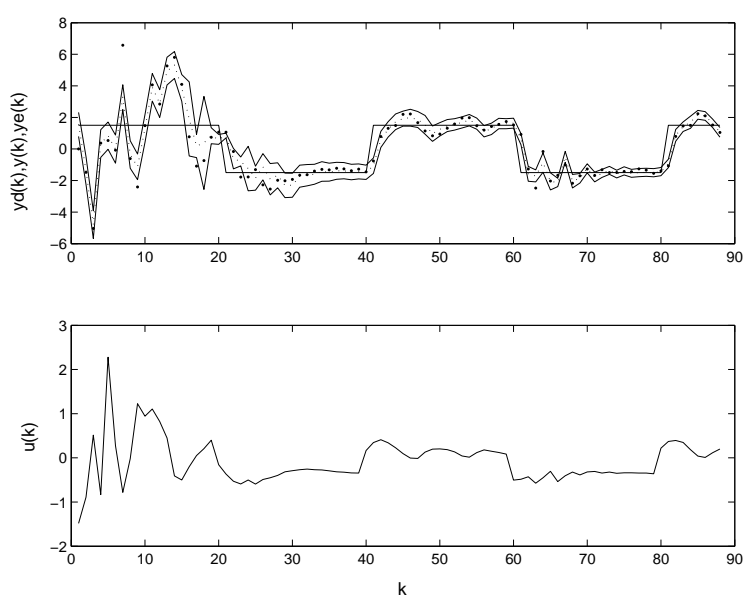

Fig. 2. Adaptive control of 1st nonlinear system with linear inverse dynamics. Control initiated from $k=10$. The upper plot shows the expected output $y_{e} \pm 2$ standard deviations.

\subsection{Nonlinear system 2 - nonlinear inverse dynamics}

The second nonlinear example considers a more complex system described by the following nonlinear functions:

$$
\begin{aligned}
f(\mathbf{x}(t)) & =\frac{y(t) y(t-1) u(t-1)(y(t-2)+1)}{1+y(t-1)^{2}+y(t-2)^{2}} \\
g(\mathbf{x}(t)) & =\frac{1}{1+y(t-1)^{2}+y(t-2)^{2}}
\end{aligned}
$$

where $\mathbf{x}=[y(t) y(t-1) y(t-2) u(t-1)]^{T}$. We also assume that the set-point will always be positive. In this case, it is not possible to use the polynomial $R\left(q^{-1}\right)$ in cost function (3) to stabilize the inverse dynamic, since for different output references, it will be necessary to have different coefficients. However, by using the cost function (5) it is possible, with $\lambda=0.6$, to have a stable closed loop response for a set of set-points, as shown in figure 3. 

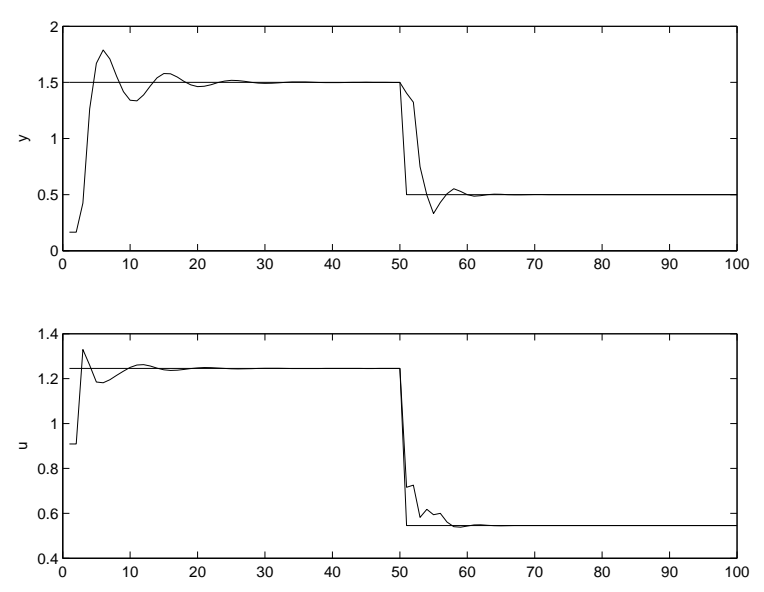

Fig. 3. Control of 2nd nonlinear system with nonlinear inverse dynamics
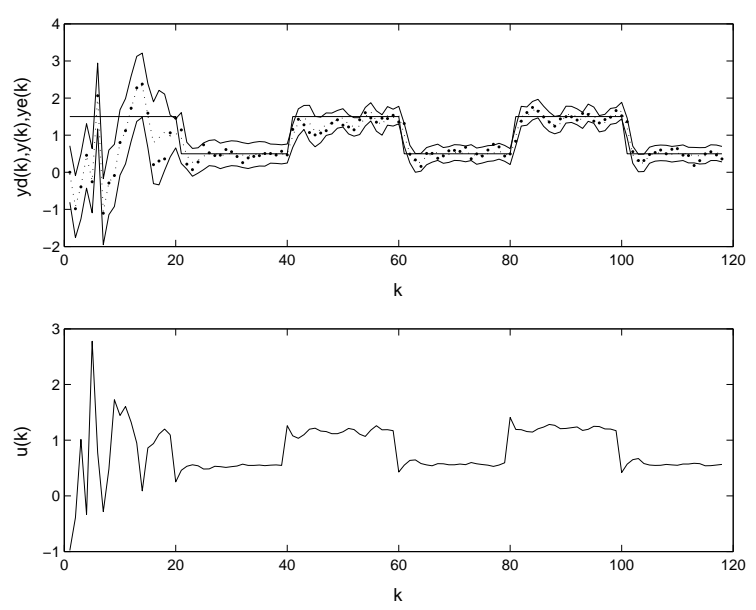

Fig. 4. Adaptive control of 2nd nonlinear system with nonlinear inverse dynamics. Control initiated from $k=10$. The upper plot shows the expected output $y_{e} \pm 2$ standard deviations.

In order to simulate the adaptive controller, we consider the system noise with a variance $\sigma_{n}^{2}=$ 0.001 , and 10 initial data points, for starting the controller. The value of $u_{d}$ was calculated from the identified model. In this case, the system takes more time to converge to the nominal response, as seen in figure 4 , since the control output depends also on the estimation of $u_{d}$. As in previous example, the control signal is bounded and well behaved.

\subsection{Nonlinear system 3 - output multiplicities}

Even though we have extended the class of system to be controlled by this simple strategy, there is still some work to be done. There are systems having output multiplicities; i.e. for fixed steady state values of the input variable, more than one value of the output variable is produced, which represent a more challenging control problem. Let us consider the following (third) nonlinear system:

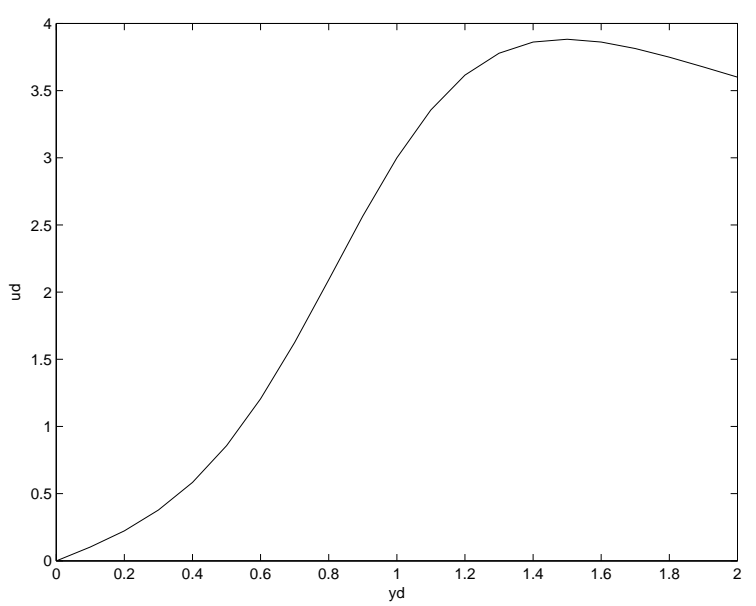

Fig. 5. Static characteristics for the third system, with output multiplicities.
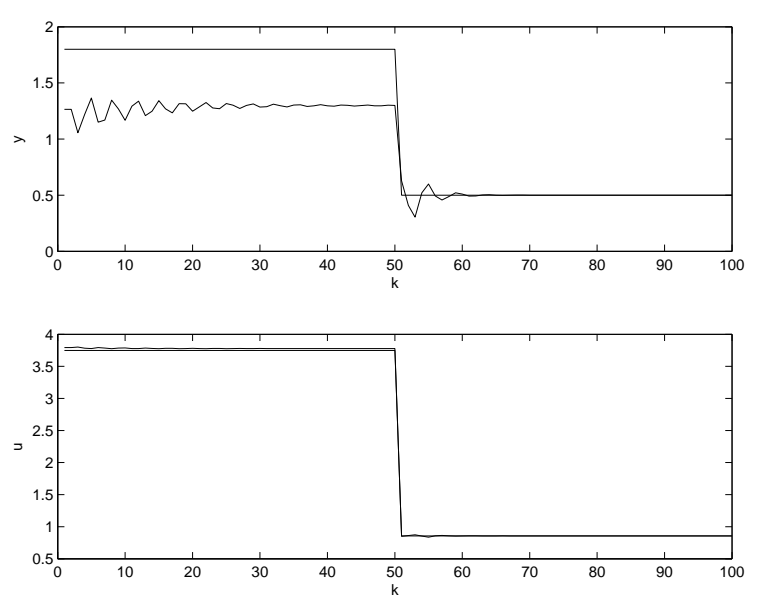

Fig. 6. Control of 3rd nonlinear system with nonlinear inverse dynamics and output multiplicities.

$f(\mathbf{x}(t))=\frac{y(t) y(t-1) y(t-2) u(t-1)(y(t-2)-1)}{1+y(t-1)^{2}+y(t-2)^{2}}$

$g(\mathbf{x}(t))=\frac{1}{1+y(t-1)^{2}+y(t-2)^{2}} ;$

As seen in figure 6 , for a given input there are two output values which can be reached by the same input value. By using the cost function (5) with $y_{d}=1.8, u_{d}=3.74$ and $\lambda=0.7$, we have a stable closed loop response but the system stabilizes at a different point, figure 6 . This result was expected, since the system static characteristic has output multiplicity, which leads to a non-unique solution for the necessary condition given by $\frac{\partial J_{2}}{\partial u(t)}=0$. Notice that the system at the desired set-point is unstable and has an unstable inverse as well, as we pointed out in section 2 the proposed approach may not work in these cases. For dealing with this type of system, further refinements are necessary, which are our current interest. 


\section{FINAL REMARKS}

This work has proposed and analyzed the advantages and limitations of a new cost function for a GMV controller, which can be used to control a broader class of nonlinear system than the conventional GMV cost function. For a system having linear inverse dynamics, the conventional GMV approach can be used. However, if the static characteristics of the process do not have output multiplicities and the inverse dynamic is nonlinear, then the approach proposed provides a simple way to control the process. The results obtained by combining the GMV controller with a nonparametric identification algorithm based on Gaussian Process priors demonstrate the viability of this approach for controlling an unknown nonlinear system. These results can also be extended to parametric models.

The use of Gaussian Process priors provides variance estimates for model predictions, which mean we no longer need make the certainty equivalence assumption. The GP also allows adaptive control, where the model complexity can adapt the degrees of freedom continuously and flexibly online, depending on the data, unlike parametric models, which require a discrete model selection process.

\section{REFERENCES}

Aoyama, A., F.J. Doyle III and V. Venkatasubramanian (1996). Control affine neural network approach for non-minimum-phase nonlinear process control. J. Proc. Control 6(1), 17-26.

Bittanti, S. and L. Piroddi (1997). Neural implementation of GMV control schemes based on affine input/output models. IEE Proc.Control Theory Appl. 144(6), 521-530.

Chen, F.C. and H. Khalil (1995). Adaptive control of a class of nonlinear discrete-time systems. IEEE. Trans. Automatic Control 40(5), 791801.

Goodwin, G., O. Rojas and H. Takata (2001). Nonlinear control via generalized feedback linearization using neural networks. Asian Journal of Control 3(2), 79-88.

Jagannathan, S. and F.L. Lewis (1996). Robust implicit self-tuning regulator: Convergence and stability. Automatica 12(32), 1629-1644.

Kocijan, J., R. Murray-Smith, C.Rasmussen and A. Girard (2004). Gaussian process model based predictive control. In: American Control Conference, Boston.

Liang, F. and H.A. ElMargahy (1994). Self-tuning neurocontrol of nonlinear systems using localized polynomial networks with CLI cells. In: Proceedings of the American Control Conference. Baltimore, Maryland. pp. 2148-2152.
Murray-Smith, R. and A. Girard (2001). Gaussian process priors with ARMA noise models. In: Proceedings of the Irish Sgnals and Systems Conference. Maynooth, Ireland. pp. 147-152.

Murray-Smith, R. and D. Sbarbaro (2002). Nonlinear adaptive control using non-paramtric gaussian process prior models. In: Proceedings of the 15th IFAC world congress. Barcelona, Spain.

Narendra, K.S. and P. Parthasarathy (1990). Identification and control of dynamical systems using neural networks. IEEE. Trans. Neural Networks 1(1), 4-27.

Portier, B. and A. Oulidi (2000). Nonparametric estimation and adaptive control of functional autoregressive models. SIAM J. Control Optm. 39(2), 411-432.

Seeger, M., C Williams and D.W. Lawrence (2003). Fast forward selection to speed up sparce Gaussian process regression. In: Proceedings of the Ninth International Workshop on AI and Statistics.

Solak, E., R. Murray-Smith, W. E. Leithead, D. J. Leith and C. E. Rasmussen (2003). Derivative observations in Gaussian process models of dynamic systems. In: Advances in Neural Information Processing Systems 15 (S. Thrun S. Becker and K. Obermayer, Eds.). pp. 10331040. MIT Press. Cambridge, MA.

Talebi, H.A., R.V. Patel and K.Khorasani (2000). A neural network controller for discrete time nonlinear non-minimum phase system. In: IJCNN 2000, Proceedings of the IEEE-INNSENNS International Joint Conference on Neural Networks. Vol. 4. July. pp. $311-316$.

Williams, C.K. (1998). Prediction with Gaussian process: From linear regression to linear prediction and beyond. In: Learning and Inference in Graphical Models (M.I. Jordan, Ed.). Kluwer. pp. 599-621.

Ying, H. (1999). Analytical analysis and fedback linearizing tracking control of a general Takagi-Sugeno fuzzy dynamic system. IEEE Transaction on System, Man and Cybernetics- Part C 29(1), 290-297.

Yue, H., T. Chai and C. Shao (1997). Adaptive control of nonlinear non-minimum phase sytems using neural networks. In: 36th $C D C$. San Diego, California USA, December.

Zhu, Q. and L. Guo (2004). Stable adaptive neurocontrol for nonlinear discrete-time systems. IEEE. Trans. Neural Networks 15(3), 653662.

Zhu, Q.M., Z. Ma and K. Warwick (1999). Neural network enhanced generalized minimum variance self-tuning controller for nonlinear discrete-time systems. IEE Proc.-Control Theory Appl. 146(4), 319-326. 